\title{
Vinculación del Instituto Torroja a lo largo de su historia con la investigación en materiales cerámicos y vítreos
}

\author{
J. Ma. RINCÓN y M. ROMERO \\ Grupo / Lab de Materiales Vítreos y Cerámicos, IETcC, CSIC. \\ Madrid
}

\begin{abstract}
El Instituto de Ciencias de la Construcción Eduardo Torroja (IETcc) desde su fundación no sólo ha realizado a lo largo de su historia investigaciones y valiosas aportaciones científicas y técnicas en el campo de los materiales cerámicos y vidrios, sino que además fue uno de los principales fundadores de la actual Sociedad Española de Cerámica y Vidrio (SECV)con la destacada participación de Francisco Arredondo y Verdú. En los últimos años esta línea de investigación se ha visto reforzada con la creación del Grupo y Laboratorio de Materiales Vítreos y Cerámicos, por lo que se realiza una revisión de la relación de este centro con este tipo de materiales recordándose los investigadores y publicaciones que más destacaron en este campo
\end{abstract}

Palabra clave: Actividad de I+ D, materiales cerámicos, vidrios, construcción

\section{The Torroja Institute historical links with ceramic and y glass research}

Ever since its inception and throughout its history, the Eduardo Torroja Institute for Construction Science of the CSIC (IETcc, CSIC) has conducted research in and made valuable scientific and technical contributions to the area of ceramic and glassy materials, and played a key role in the founding of the present Spanish Ceramic and Glass Society (SECV), under the leadership of Francisco Arredondo y Verdú. The creation in recent years of the Glassy and Ceramic Materials Group and Laboratory has reinforced this line of research. The present paper reviews the effort deployed by the Institute in connection with this type of materials, with a tribute to some of the most prominent publications in the field and their authors.

Key words: $R+D$ activities, ceramics, glasses, construction

\section{INTRODUCCIÓN}

La primera rama de actividades por su aportación al Producto Interior Bruto en España, corresponde al deMateriales de Construcción y dentro del mismo en un gran porcentaje al de los Vidrios y Materiales Cerámicos (1). Nuestro país dispone de un importante sector de industrias, tanto en Obras Públicas como en Vivienda con una posición preeminente en la producción de materiales básicos en la Construcción. Habiendo ya superado defectos de tipo estructural así como de mentalidad, es el sector de la Construcción y sus materiales en estos momentos uno de los más innovadores de la economía española, incorporando una amplia gama de productos que resultan de la combinación de otros (materiales compuestos) lo que constituye la tendencia más señalada del momento actual.

\section{UN PASADO YA HISTÓRICO}

El Instituto Eduardo Torroja ("El Torroja" en adelante, como se le conoce coloquialmente en los medios científicos y empresariales españoles) recientemente celebró el 100 aniversario del nacimiento de su fundador: D. Eduardo Torroja. Su evolución eatá marcada por los siguientes hitos: a) Fundación en 1934 del Instituto Técnico de la Construcción y Edificación,

b) entre 1941- 46 adhesión del citado Instituto al CSIC dentro del entonces Patronato Juan de la Cierva,

c) consolidándose como centro de investigación en la calle Medinaceli 4 de Madrid entre 1946- 48 (2).
Es un hecho que desde su fundación ha estado íntimamente vinculado con los materiales cerámicos y vítreos, como materiales de construcción que son y usados tanto como materiales estructurales de fábrica de edificios o en su más abundante uso como materiales de revestimiento y de pavimento en la construcción.

Ya Eduardo Torroja, fundador de este centro de investigación, en su conocida obra "Razón y Ser"(3), señalaba la importancia que se merece esta amplia gama de materiales como "esenciales en cualquier obra arquitectónica y de ingeniería civil u obras públicas". De hecho, en el edificio del IETcc, construido en 1951 y que recientemente ha obtenido un nivel I de protección en la catalogación de edificios singulares por parte del Ayuntamiento de Madrid, existen elementos cerámicos entre los que destacan su fachadas de ladrillo rojo. Eduardo Torroja entendía que "...la técnica de la construcción no progresaría si no se desarrollaban nuevos materiales o se mejoraban las propiedades de los existentes" ... y que "en el arte de la construcción existe siempre un fondo esencialmente científico..." (discurso de ingreso de ET en la Acad. de Ciencias en 1944) (3). Lo que merece la pena recordar a propósito de las polémicas surgidas en años posteriores precisamente en este centro sobre los binomios: Investigación - Asistencia Técnica, Ciencia- Tecnología o el que está aun más descompensado: Ciencia + Tecnología + Normativa (4).

Además dela multidisciplinaridad abiertamente reconocida : “...Eduardo Torroja demostró que es posible crear y apoyar centros multidisciplinares que integren en sí mismos las cuatro facetas: ciencia- técnica- docencia- empresa..." (M. C. Andrade 
en: Ciencia, Ingeniería Empresa, El País, suplemento La Cultura del 27 agosto 1999, pag. 30), creemos que es necesario ampliar este concepto extendiendo esta multidisciplinaridad no sólo a las distintas disciplinas científicas, sino también a la cada vez más amplia gama de materiales que "visten y estructuran una construccion... (" técnica plures, opera unica") y nosotros diríamos : DIVERSA ELEMENTA à UNUM OPUS FACIUNT. Según Martin Heidegger (1956): “ las casas no se construyen para que resistan sino para habitar. Y se construye y habita para vivir" (Barañano en: Eduardo Torroja, EL CULTURAL, 21.11.99.pag. 25 ). Los materiales ceramicos, pues, "nos envuelven" y "presentan la cara" mejorando la habitabilidad y el confort de nuestra vida diaria allí en donde nos encontremos. Como anécdota es bueno recordar que el ladrillo por ejemplo, fué la primera patente o sello de obra de Miguel Fisac, un arquitecto muy ligado por diversas razones a nuestro Instituto y que defendió siempre a este material demostrando las posibilidades del mismo: “...el ladrillo visto tiene un carácter escultórico mas metafísico que físico (E. Dominguez Uceta, en: Peaton sediento frente al muro de ladrillo, La Mirada del Arquitecto, Diario El Mundo...,1998 ). Así pues, el Instituto Torroja ha estado siempre comprometido desde sus inicios con la Ciencia y Tecnología de los materiales cerámicos y vítreos como vamos a ver sucintamente a continuación.

\subsection{RELACIONES DEL IETCC CON LA SOCIEDAD ESPAÑOLA DE CERÁMICA Y VIDRIO (SECV)}

La SECV fué fundada el 16 de febrero de 1960 bajo la inspiración principal del Prof. Antonio García Verduch del entonces Depto. de Silicatos del Inst ${ }^{\circ}$. de Edafología del CSIC, aunque ya se mantenían actividades en este campo desde 1958 como a reseñado el Prof. Verduch recientemente con todo detalle en un muy reciente artículo (5). En el mismo se expone con todo detalle la creación de la SECV en cuya redacción de Estatutos y acta de constitución participó de manera destacada: Francisco Arredondo y Verdú, miembro del Inst ${ }^{\circ}$. Torroja, conjuntamente con Vicente Aleixandre Ferrandis, Antonio García Verduch y ocho miembros más de la Comsión Organizadora, constituyéndose la SECV el 16 de Febrero de 1960 en el Salón de Actos del entonces Patronato Juan de la Cierva. En dicha reunión Francisco Arredondo estuvo en la mesa presidencial conjuntamente con Vicente Aleixandre y Antonio García Verduch, representando a toda la comisión constitutiva de la SECV.

En junio de 1960 se organizó en Madrid la I Reunión General Técnica de la SECV con el nombre de I Semana de Estudios Cerámicos con asistencia de unas 40 personas, entre ellos varios investigadores del Torroja. La V Semana de Estudios Cerámicos que tuvo lugar en 1963 entre el 7-10 mayo de dicho año se celebró en la sede principal del IETcc, CSIC. Francisco Arredondo estuvo en la Junta Directiva de la SECV entre 1961 y hasta mayo/ junio de 1974 figurando como tal en las correspondientes números del Boletín de la SECV del que formó parte en la misma época del Comité de Redacción. Destacados investigadores del Instituto Torroja, tales como: el mismo Arredondo, Francisco Soria, Demetrio Gaspar y Aurelio Alamán, contribuyeron con sus comunicaciones a las Semanas de Estudios Cerámicos, que fueron el germen del actual Congreso Nacional de Cerámica y Vidrio (6)

\section{ETAPAS DE LA INVESTIGACIÓN EN MATERIALES CERÁMICOS Y VIDRIOS EN EL IETCC}

Como es habitual en todo el CSIC, cada periodo de la vida de sus centros ha llevado el sello de la especialidad e intereses de cada uno de los investigadores que han protagonizado en este centro de investigación el liderazgo de investigación en estos materiales. Después de recopilar todos los datos disponibles la actividad en materiales vítreos y cerámicos en el Torroja ha estado marcada por las siguientes etapas:

Primera Etapa: Anteriormente a la creación de la SECV ya existía interés y actividad respecto a los materiales cerámicos en el Torroja. La primera publicación en este centro de investigación sobre estos materiales corresponde al año 1951 escrita por Demetrio Gaspar y titulada: "Arcillas y Ladrillos". En años posteriores Fernando Casinello, Dr. Arquitecto, que llegó a ser director del IETcc dedicó una atención especial al uso del ladrillo en la construcción con sus obras: "El Ladrillo y sus fábricas" (1958); "Arcos de ladrillo" (1958); "Los amigos del ladrillo" (1960); “Bóvedas y cúpulas de ladrillo" (1964), "Muros de carga de fabrica de ladrillo" (1964), todos ellos publicados como Monografías del Departamento de Publicaciones del Torroja. Un buen ejemplo como obra constructiva de ladrillo del arquitecto F. Casinello es la Urbanización Gran Habitat en Arganda del Rey, Madrid.

En años posteriores Francisco Soria, también del IETcc, dedicó varias publicaciones a los materiales refractarios en los hornos de fabricación del clinker (7).

Segunda Etapa: Francisco Arredondo, Dr. Ingeniero de Caminos nacido en 1920 y fallecido en 1996, empezó a trabajar en el IETcc en el año 1948 habiendo fundado y dirigido el Departamento de Materiales (en 1954) siendo su jefe hasta que en 1970 pasó a Director del Instituto, cargo en el que estuvo hasta 1980. Dentro de este Departamento se creó la Sección de Cerámica que investigaba tanto materiales de arcilla cocida como materiales refractarios llevando a cabo numerosa asistencia técnica en patologías de bovedillas, tejas, baldosas, gres, sanitarios, etc... Se publicó un libro monográfico dedicado a los Materiales Cerámicos en la Construcción (8) y entre los años 1968- 1974 se desarrolló una intensa actividad relacionada con estos materiales también en cuanto a cursos de especialización y todo tipo de investigaciones entre las que cabe destacar las dedicadas a los efectos de la porosidad en las propiedades tecnológicas de los productos cerámicos para la construcción, se apoyó la fundación de la SECV, se participó en el Grupo de Trabajo Comité Técnico no 67 de Cerámica. Destacan además las colaboraciones en materiales cerámicos de sanitarios con la compañía ROCA e incluso en el año 1972 se fabricaron 400 ladrillos para la Feria Monográfica de Cerámica y Elementos Decorativos de Valencia. Las referencias debidas a contribuciones de Francisco Arredondo son numerosas recogiéndose las principales agrupadas en la referencia (9).

En años posteriores la actividad en Materiales Cerámicos de la Construcción fue notable, de tal manera que Enrique Gippini con su libro, "Contribución al estado de la composición de las pastas cerámicas" (editado en 1969 y hoy totalmente agotado), que recoge su trabajo de Tesis Doctoral, ha sido de los más solicitados. Posteriormente, en 1974 edita el libro: "Pastas Cerámicas", que tuvo también una gran demanda y fué editado por la SECV, constituye una bien conocida y obligada referencia para todos aquellos técnicos e investigadores que trabajan con los materiales cerámicos (10). Gippini desarrolló su actividad investigadora en el Torroja entre los años 19651970 incidiendo en el comportamiento de barbotinas y su reología. Este investigador publicó además en 1973 la obra: "Seis procedimientos de ensayo y una norma de calidad para bovedillas cerámicas", que hoy en día aun sigue teniendo vigencia.

Son numerosas las citas dedicadas a los materiales cerámicos y vidrios en las dos revistas que edita el Instituto con el patrocinio del CSIC: MATERIALES DE CONSTRUCCIÓN , que recientemente celebró su 50 aniversario (11) (se edita desde 1951 ininterrumpidamente) editó un número monográfico editado para esta celebración en la que tampoco faltaron aportaciones del campo de la ciencia y la tecnología de los materiales que nos ocupan. En el caso de 
INFORMES DE LA CONSTRUCCIÓN (que se edita desde 1948 ininterrumpidamente) hemos recogido las siguientes referencias pertenecientes sobre todo a los primeros números de esta revista: "Ventanas de un edificio destinado a oficinas en Río de Janeiro" (N. 3, 1948); “Construcciones armadas de ladrillo" y "El concepto actual de los productos vitrificados" por P. de Goote ( $\mathrm{N}^{\mathrm{o}} 4$, 1948); "Construcciones con piezas de cerámica armada" por H. Gronholen (N. 11, 1949); “El horno- túnel en la industria azulejera" por E,. Tovar ( $\mathrm{N}^{\circ} .39$, 1952); "Eflorescencias en ladrillos " por D. Gaspar (No. 46, 1952); “Un muro de ladrillo" por M. Fisac (No. 47, 1953); “La arcilla, materia prima para materiales de construcción", por M. Lepingle (No: 62, 1954) y “Contribución al estudio de la destrucción química del refractario del horno de cemento" por Francisco Soria $\left(N^{a} .63,1954\right)$.

Tercera Etapa: Antes de lo que hemos considerado como Tercera Etapa, existe un largo periodo de "desencuentro" entre los materiales cerámicos y el Torroja. Hasta el año 1986 en que Gloria Fernández- Arroyo investigadora del Instituto de Cerámica y Vidrio, también del CSIC, se incorpora al Torroja, apenas hay actividad investigadora de relieve relacionada con este tipo de materiales. Aun así se siguen realizando informes técnicos y expedientes relacionados con los mismos (sobre todo por Bernardo Bacle). Gloria Fernández- Arroyo (19861993) aporta por primera vez a este centro de investigación el interés por el vidrio como tal, debido a sus investigaciones sobre vidrios con aplicaciones en Energía Solar (12).

Coincide también con esta etapa la dedicación de otro investigador del Instituto, Cesar del Olmo, a los temas de colocación de pavimentos y revestimientos cerámicos llegando a ser un único y valioso especialista en cementoscola para la colocación de pavimentos, un tema que tiene un interés relevante para todo el sector, especialmente el del área de Castellón, que ha manifestado repetidas veces la necesidad de que el Torroja dedique investigaciones a este tema.

Cuarta Etapa: Se inicia con la llegada en 1994 de Jesús Ma. Rincón al IETcc, después de haber desempeñado una intensa actividad investigadora durante 25 años en el Instituto de Cerámica y Vidrio, que precisamente es la sede de la SECV de la que fue su Secretario General en el periodo 1988-92 (13). Esta etapa se consolida con la creación del Grupo y Laboratorio de Materiales Vítreos y Cerámicos que cuenta actualmente con tres personas en plantilla (Prof. Rincón, Dr. Maximina Romero, CT y Pilar Díaz, técnico de la escala laboral del CSIC), así como dos becarios (uno pre- y otro postdoctoral) y la visita intermitente desde el año citado hasta la fecha de unos 2- 3 investigadores extranjeros por año.

\section{UN PRESENTE MUY ACTIVO}

Esta "pequeña historia" de los materiales cerámicos en nuestro Instituto ha estado, pues, lamentablemente jalonada de una serie de "encuentros y desencuentros", para concluir desde hace ya once años en que los Materiales Cerámicos y Vidrios en la Construcción han "reencontrado" de nuevo su camino en este reconocido centro de investigación en un contexto íntimamente relacionado con el sector productivo español y europeo. $Y$ todo ello debido a las investigaciones que actualmente se llevan a cabo en el Grupo/ Lab/. de Materiales Vítreos y Cerámicos del IEtcc. Materiales vítreos y cerámicos, que abarcan los siguientes campos: a) Productos de la arcilla; b) optimización del diseño de productos cerámicos: El nuevo producto LIGERAMICA $^{\circledR}$ desarrollado bajo contrato con la compañía CERAMICAS DIAGO consiguió el ALFA DE ORO de la Feria CEVISAMA en el año 2000; c) investigación sobre ladrillo altamente sinterizados tipo klinker; d) Experiencias en patología en materiales cerámicos y vidrios; e) Durante estos años se han elaborado además numerosos expedientes y dictámenes sobre patología de los materiales vítreos y cerámicos: Ladrillos (bovedillas y ladrillos antiguos...), tejas, gres catalán, gres rústico, pavimentos y revestimientos cerámicos vitrificados... todos ellos bajo contrato con relevantes industrias del sector (14). La presencia del Torroja por medio de ese grupo de Investigación ha sido ininterrumpida en la Feria CEVISAMA desde el año 1994.

La investigación en Vidrios y Materiales Cerámicos de la Construcción está, pues, actualmente activa en este centro por la intensa actividad desarrollada en los últimos años, sobre todo como consecuencia de las necesidades y demandas de este estratégico sector industrial. Se imparten sobre este tipo de materiales (cursos CEMCO, FOCIMATT...y más recientemente uno de Doctorado en la Universidad Carlos III de Madrid) incluido en el programa de Ciencia y Tecnología de Materiales habiéndose editado varios libros monográficos, algunos de ellos con editoriales científicas de otros países (15-16).

En años más recientes se han incorporado a estas investigaciones los materiales de piedra natural con la idea de englobar en un misma disciplina o "cuerpo de doctrina" los materiales cerámicos, vítreos y pétreos como Materiales Inorgánicos en la Construcción, con un enfoque unitario temático que hasta ahora no existía en nuestro país.

\section{UN FUTURO A CONSOLIDAR Y NECESITADO DE APOYO PORQUE LO DEMANDA EL SECTOR}

Por tanto, la investigación en materiales cerámicos y vidrios en la construcción podría, por fin, ser algo "estable" en este centro de investigación, al menos para estas primeras décadas del siglo XXI. Pero para ello, hay que continuar el esfuerzo emprendido y seguir contando como hasta ahora con el apoyo del sector industrial (tanto fabricantes como constructores) apostando por líneas de futuro tales como: El desarrollo de nuevos materiales de tipo compuesto (ó "composites"); la profundización en la optimización del diseño y propiedades mecánicas con minimización de masa y el abordaje de nuevas técnicas de caracterización microestructural y analítica, en la que los componentes del Grupo son expertos, que permita mantener e incrementar el nivel científico alcanzado.

\section{AGRADECIMIENTOS}

A todos aquellos miembros presentes y pasados del Inst ${ }^{\circ}$. Torroja que han aportado datos y experiencias propias de "la pequeña historia" de los materiales cerámicos dentro de la ya larga vida de este centro de investigación y todos aquellos que colaboraron de una manera $\mathrm{u}$ otra en este tipo de investigaciones dentro de este centro.

\section{BIBLIOGRAFÍA}

1. E. Criado, E. Sánchez, M. Regueiro, “La industria cerámica española ante un cambio de ciclo?", Bol. Soc. Esp. Ceram. V. 43 (1) 85-101 (2004).

2. F. Arredondo, C. Benito, G. Echegaray, J. Nadal, A. Paez y F. Del Pozo, “La Obra de Eduardo Torroja", Colección Cultura y Ciencia, Ed. Instituto de Empresa, Madrid, 1977.

3. E. Torroja Miret, "Razón y Ser", Textos Universitarios, Ed. IETcc, CSIC, $7^{\mathrm{a}}$. Edición, Madrid, 1991.

4. Colectivo Beta, “La reestructuración del Inst ${ }^{\circ}$. E. Torroja de la Construcción y del Cemento: No fue posible el futuro, pp: 122- 139, en: Política Científica y Futuro del CSIC, Ed. API, Madrid 1984 en: Política Científica y Futuro del CSIC.

5. A. García- Verduch, "Sociedad Española de Cerámica y Vidrio. Sus primeros pasos." Bol. Soc. Esp. Ceram. V. 41 [4] 415-421 (2002).

6. Varios autores, "I , II y III Semanas de Estudios Cerámicos", Ed. SECV, Madrid, 1961,1963, 1965; a saber:

a. D. Gaspar, La plasticidad de pastas cerámicas, $1{ }^{\text {a }}$ Semana de Estudios Cerámicos, 173-183,1961.

b. F. Arredondo, “Desecación de productos de arcilla", $1^{\text {a }}$ Semana de Estudios Cerámicos, 91-101,1961.

c. A. Alemán, "Permeabilidad al agua de muros de ladrillo", Semana de Estudios Cerámicos, 89- 100, 1965. 
d. F. Arredondo, "Hormigón de arcillas dilatadas", 3 a Semana de Estudios Cerámicos, 1- 15, 1965.

7. F. Soria, “Físicoquímica del sistema refractario - clinker en los hornos de cemento", $2^{\text {a }}$ Semana de estudios Cerámicos, 121-131, 1963.

8. F. Arredondo, "Materiales Cerámicos y vidrio", en: Serie Estudio de Materiales, Tomo VI, Ed. IETcc, CSIC, 1963, 1ª Edición, en 1980 se editó la $9^{a}$ Edición.

9.F. Arredondo: "Fabricación de ladrillos", Madrid ,1951; “La resistencia de los muros de ladrillos", Madrid, 1956; "Los defectos de las obras de ladrillo", conferencia dada en Brasil en 1959; "La alianza ceámica- acerohormigón" , conferencia en Uruguay ,1959.

10. E Gippini "Pastas Cerámicas", Ed. IETCC, CSIC 1979.

11. J. Calleja, “Cincuenta años de la revista : Ultimos Avances en Materiales de Construcción", Materiales de Construcción 51(263-264) 5-25 (2001).

12. G. Fernández Arroyo, "El Vidrio", Monografías Torroja, Ed. IETcc, CSIC, 1990

13. J. Ma. Rincón, “La Sociedad Española de Cerámica y Vidrio y su labor a lo largo de más de 25 años", ALMACEN de Materiales de Construcción (4) 29-33 (1991).

14. Varios autores, “El Vidrio en la Construcción, Materiales de Construcción, 4 6,1996.

15. T. Manfredini, J. C. Pellacani and J. Ma. Rincón, Glass- Ceramics: Fundamentals and Applications, Ed. Mucchi Editore, Módena, Italia 1998 16. J. Ma. Rincón and M. Romero, "Characterization Techniques of Glasses and Ceramics", Springer- Verlag, 1999.

17. J. Ma. Rincón, M. Romero, M. Jordan y J. P. Gutierrez, "Materiales Inorgánicos en la Construcción para el siglo XXI", Ed. IETcc, CSIC y Univ. Miguel Hernández, Elche, 2001.

Recibido: 30.07 .04

Aceptado: 10.01 .05

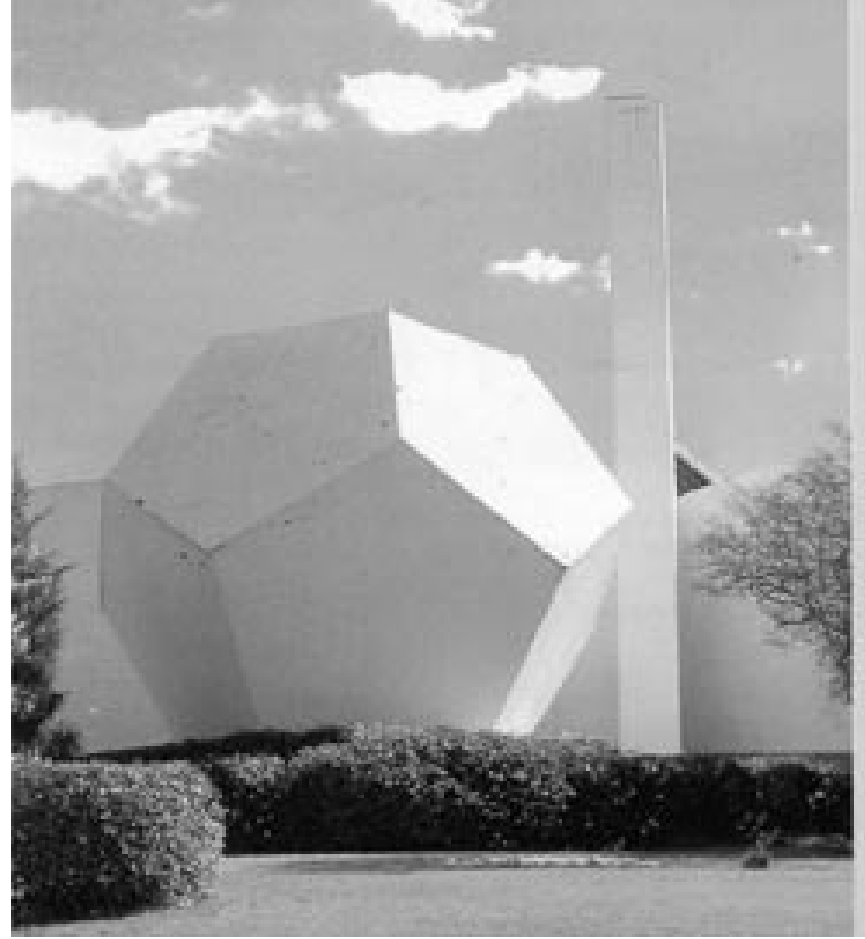

Fig. 1.- Depósito de carbón del Instituto Torroja, que se ha constituído en el símbolo de este centro de investigación del CSIC.


Fig. 2.- Lámina de hormigón pretensado denominada "el elefante" en el patio de Alarifes del Torroja 